\title{
Epidemia de sobredosis relacionada con la prescripción de analgésicos opioides en Estados Unidos
}

\section{Overdose epidemic linked to the prescription of opioid analgesics in the United States}

\author{
Dr. Josep Guardia Serecigni*. \\ * Vicepresidente de Socidrogalcohol. Doctor en medicina. Consultor senior de psiquiatría, Unidad de Conductas Adictivas, \\ Servicio de Psiquiatría. Hospital de la Santa Creu y Sant Pau, Barcelona, España.
}

$\mathrm{H}$ asta los años 80 los médicos eran más prudentes respecto a la prescripción de opioides para el tratamiento del dolor, en pacientes que no fueran terminales, dado que conocían su riesgo adictivo.

Sin embargo, una campaña de sensibilización a los médicos de Estados Unidos, con difusión de evidencias de baja calidad sobre la supuesta eficacia y seguridad de los analgésicos opioides, para el tratamiento del dolor, generó un efecto de prescripción indiscriminada e incluso de mayor demanda de analgésicos opioides por parte de los pacientes.

Una carta de un solo párrafo de Porter y Jick publicada en el New England Journal of Medecine en 1980, refirió que de 39.946 pacientes hospitalizados, 11.882 recibieron la prescripción de un opioide. Que sólo cuatro de ellos desarrollaron adicción y sólo en uno de ellos la adicción fue considerada como grave. La carta acababa diciendo que el desarrollo de adicción es poco frecuente en los pacientes que no tienen antecedentes de adicción.

Esta carta ha sido citada 608 veces durante años, el $72,2 \%$ de la citas la consideraron como evidencia de que la adicción a opioides es poco frecuente y el 80,8\% no mencionaron que la muestra era de pacientes hospitalizados. El mensaje que el riesgo de adicción a los analgésicos opioides era pequeño tuvo una gran difusión y puede haber contribuido a la epidemia de analgésicos opioides en Norteamérica. (Brauser, 2017; Leung, Macdonald, Stanbrook, Dhalla y Jauurlink, 2017).
Una mayor disponibilidad de opioides entre la población generó una gran expansión de su consumo, con un rápido incremento de las tasas de abuso, adicción y sobredosis. Cuando intentaron medidas de contención de la epidemia algunas personas pasaron a tomar opioides ilegales como heroína o fentanilo y sus derivados. El incremento de muertes por sobredosis ha movilizado al gobierno de los Estados Unidos para reducir su prescripción y aumentar la oferta de tratamiento a las personas que han desarrollado adicción y sobredosis (CDC, 2016; Madras, 2017).

Durante las dos últimas décadas, las ventas de opioides por prescripción se han incrementado en un $300 \%$ y más del $50 \%$ han sido prescritas para tratamiento del dolor crónico no canceroso. Casi la mitad de personas que se han incorporado a tratamiento, por adicción a opioides, han referido que su primer contacto fue por prescripción médica para tratamiento del dolor y el $80 \%$ de los que presentan adicción a la heroína pasaron por una etapa previa de abuso de analgésicos opioides (Madras, 2017).

Se calcula que un $30 \%$ de estadounidenses sufren algún tipo de dolor, la mitad de ellos diariamente y en una tercera parte el dolor se considera como grave, siendo la lumbalgia y la osteoartritis las formas más frecuentes de dolor crónico. El 20\% reciben una prescripción de analgésicos opioides, sin embargo, sólo producen una reducción de 8-12 puntos sobre 100 en las lumbalgias y la tasa de abandonos de tratamiento, debido a falta de eficacia o efectos adversos, llega al 50\%. Los autores señalan que probable- 
mente se están prescribiendo con excesiva frecuencia o duración, para el dolor agudo, y que su eficacia a largo plazo, para el dolor crónico, es limitada (Lin y Vega, 2016).

Entre 1999 y 2014 se ha cuadruplicado el número de muertes por sobredosis en Estados Unidos. El $61 \%$ de ellas están relacionadas con opioides y el rápido incremento de dichas muertes -en 2014 y 2015- está relacionado con opioides sintéticos como fentanilo en el $72,2 \%$ de los casos y con heroína en el 20,6\% (Rudd, Seth, David y Scholl, 2016; NIDA, 2017).

El Centro de Prevención y Control de Enfermedades (CDC) de Estados Unidos afirma que un 20\% de las personas que reciben una prescripción inicial para 10 días, de analgésicos opioides, seguirá tomando dichos fármacos más allá de un año, lo cual sugiere su capacidad adictiva. Y además reconocen que el consumo simultáneo de analgésicos opioides y benzodiazepinas (BZD) cuadruplica el riesgo de muerte por sobredosis, en comparación con el consumo de opioides sin BZD (CDC, 2016).

\section{La Adicción a Opioides}

El dilema de los analgésicos opioides es que son medicamentos imprescindibles para aliviar determinados tipos de dolor, pero al mismo tiempo pueden llegar a causar un gran sufrimiento a las personas que desarrollan adicción y/o sobredosis.

La estimulación suprafisiológica repetida del sistema dopaminérgico, producida por el consumo continuado de opioides, puede inducir cambios en la plasticidad del cerebro (a nivel del sistema glutamatérgico en los circuitos que enlazan la corteza prefrontal con el estriado), lo cual resulta en una disminución del control inhibitorio sobre la conducta de búsqueda y consumo de sustancias, que puede llegar a ser compulsiva (Kalivas y Volkow, 2005) y que conocemos como adicción.

Los opioides tienen además un doble efecto reforzador, positivo y negativo. El positivo es debido al efecto euforizante y de recompensa cerebral. El negativo es consecuencia de su efecto de alivio del dolor, no sólo corporal sino también el de tipo emocional o psíquico, causado por acontecimientos estresantes o traumáticos. Por este motivo, las personas que sufren un trastorno mental obtienen un efecto reforzador negativo más poderoso y ello les convierte en más vulnerables hacia la adicción a opioides (Guardia, Surkov y Cardús, 2010).

$\mathrm{El}$ riesgo de adicción aumenta también cuando se utilizan de manera diferente a la prescripción hecha por el médico, ya sea a dosis más elevadas o bien usando una vía de administración más directa que la vía oral, o también cuando se consumen asociados a otros fármacos o al alcohol, dado que se producen peligrosas sinergias, con elevado riesgo de sobredosis, al asociar unos a otros (NIDA, 2012).

\section{Epidemiología de consumo de analgésicos opioides}

Estados Unidos ha sufrido una epidemia de sobredosis de opioides. Cada día mueren 91 personas por sobredosis en este país, bien sea por analgésicos opioides prescritos o bien por opioides ilegales. En 2016 se han producido más de 64.000 muertes por sobredosis, que incluyen más de 15.000 por heroína y más de 20.000 por opioides sintéticos. El aumento de muertes por sobredosis que se ha producido en la última década, se ha acelerado en los últimos años y parece que puede repercutir incluso en una reducción de la expectativa de vida de la población de Estados Unidos (NIDA, 2017).

Este abuso de opioides supone un coste de 20 billones de dólares al año debido a gastos de los servicios de urgencias y cuidados hospitalarios. Todo ello a pesar de que no existe evidencia científica de que dichos fármacos sean eficaces para el tratamiento del dolor crónico y que pueden producir además graves consecuencias negativas, como adicción, sobredosis, caídas y lesiones por accidentes (Case y Deaton, 2015).

Cada vez con mayor frecuencia la heroína se vende adulterada con fentanilo o sus derivados, en el mercado negro, sin que el comprador lo sepa. El riesgo de muerte por sobredosis es por tanto mucho mayor, ya que fentanilo y sus derivados pueden paralizar bruscamente los centros respiratorios del cerebro (EMCDDA, 2017).

Fentanilo es un opioide sintético que tiene 50 a 100 veces mayor potencia que la morfina. Los derivados del fentanilo, como acetilfentanilo, fluranilfentanilo, sufentanilo, alfentanilo, remifentanilo, carfentanilo, 3-metilfentanilo, acrilfentanilo, butirilfentanilo, parafluororfentanilo y otros, pueden llegar a tener una potencia cien veces mayor que el propio fentanilo. Concretamente carfentanilo es 10.000 veces más potente que morfina y se utiliza para producir inmovilización y capturar o anestesiar grandes animales (O'Donnell, Halpin, Mattson, Goldberger y Gladen, 2017).

En las muertes por sobredosis pueden aparecer restos de varios derivados del fentanilo en una misma persona. En los estados orientales de Estados Unidos, la mitad de muertos por sobredosis de opioides dieron positivo para fentanilo y casi la mitad de sobredosis por fentanilo o derivados no dieron positivo para otros opioides ilegales, lo cual sugiere que fentanilo y derivados podrían estar emergiendo como drogas ilícitas por ellas mismas. Dado que son muy potentes y de efecto muy rápido, la pérdida de conciencia y la muerte son casi instantáneas y requieren un tratamiento inmediato con dosis elevadas o repetidas de naloxona. Además, en una de cada cinco muertes por fentanilo y derivados no había evidencia de inyección y, por tanto, habían sido administrados por otras vías. De hecho, circulan falsos comprimidos de analgésicos opioides que contienen fentanilo o derivados (O'Donnell et al., 2017). 
El número de muertes por sobredosis de opioides de prescripción se ha cuadruplicado desde 1999 y desde 2007 supera el de muertes por sobredosis de heroína y cocaína juntas. Más del $77 \%$ de los adolescentes que han consumido heroína habían tomado previamente analgésicos opioides (vicodín, percocet u oxicontín), por tanto, estos fármacos podrían ser considerados como droga de entrada a la heroína, para los adolescentes de Estados Unidos. Los analgésicos opioides que con mayor frecuencia son objeto de abuso son hidrocodona (vicodina) -uno de cada 12- y oxicodona (oxycontín) -uno de cada 20- (NIDA, 2017).

En Europa se han detectado también muertes por sobredosis como consecuencia de la administración de potentes derivados del fentanilo en personas con antecedentes de consumo de heroína (Hikin, Smith, Ringland, Hudson y Morley, 2017), así como también un incremento de notificaciones de intoxicaciones no fatales por opioides sintéticos potentes como fentanilo y derivados (EMCDDA, 2017).

\section{Sobredosis}

Poco después de la administración de opioides se pueden presentar síntomas de somnolencia, desorientación, sedación, sudoración, miosis y un grave enlentecimiento de la respiración que puede llegar al paro respiratorio.

Cuando hablamos de sobredosis la gente piensa que deben ser de heroína y los medios de comunicación a veces refieren que son debidas a heroína de mala calidad o adulterada. Sin embargo, en Estados Unidos se producen actualmente más sobredosis por analgésicos opioides que por heroína. El riesgo de sobredosis es más elevado cuanto mayor es la pureza de la heroína y cuando va asociada a fentanilo o derivados, benzodiazepinas o alcohol.

Aunque el consumo aislado de benzodiazepinas no suele producir una depresión respiratoria clínicamente significativa, sí puede empeorar la depresión respiratoria causada por opioides y el riesgo de muerte por sobredosis aumenta sustancialmente cuando se prescriben benzodiazepinas a personas que ya toman analgésicos opioides (Horsfall y Sprague, 2016).

Jones y McAninch (2015) afirman que los fármacos de prescripción que con mayor frecuencia producen muertes por sobredosis son el consumo simultáneo de analgésicos opioides y benzodiazepinas (BZD). Según el Centro de Prevención y Control de Enfermedades (CDC) de Estados Unidos, la proporción de muertes por sobredosis de analgésicos opioides, asociados a benzodiazepinas, aumentó del 13\% en 1999 al 31\% en 2011. Entre 2006 y 2011, las muertes con implicación de BZD aumentaron un 14\% al año, mientras que las relacionadas con analgésicos opioides (sin BZD) mantuvieron las mismas cifras (Paulozzi, Jones, Marck y Rudd, 2011; Chen, Hedegaard y Warner, 2014). Y la mitad de los pacientes que sufrieron una sobredosis habían recibido la prescripción de ambos fárma- cos de un mismo prescriptor e incluso en un mismo día (Hwang, Kang, Kornegay, Staffa, Jones, y McAninch, 2016). Es decir que la co-prescripción de opioides y benzodiazepinas aumenta el riesgo de sobredosis y también de muertes por sobredosis, con un mayor riesgo para las personas que padecen alguna patología respiratoria, cardiovascular, y personas ancianas o debilitadas.

En un reciente estudio efectuado con 13.089 personas que padecían dolor crónico no canceroso, el 42,3\% habían recibido una prescripción de benzodiazepinas (BZD) en los 30 días previos a su fallecimiento (Olfson, Wall, Wang, Crystal y Blanco, 2017). Otro estudio reciente ha detectado que en el 23,5\% de los casos la prescripción de opioides y BZD fue simultánea, a pesar del efecto potenciador de las BZD sobre la depresión respiratoria inducida por opioides (Horsfall et al., 2017). Y la red de alerta de abuso de drogas (DAWN) de Estados Unidos ha confirmado también que el consumo simultáneo de opioides con benzodiazepinas o con alcohol aumenta el riesgo de sobredosis desde el $24 \%$ hasta el 55\%, en comparación con el consumo aislado de benzodiazepinas (SAMHSA, 2014).

Las benzodiazepinas podrían aumentar el riesgo de sobredosis de opioides cuatro veces más que el consumo aislado de opioides (Park, Saitz, Ganoczy, Ilgen, y Bohnert, 2015) y podrían ser una de las causas de muerte en una de cada seis muertes relacionadas con opioides (Corkery, Schifano, Ghodse y Oyefeso, 2004). Finalmente, los riesgos de la co-prescripción de opioides y benzodiazepinas se confirman por el reciente incremento de la prevalencia de dicha co-prescripción (Hwang et al., 2016) y por un desproporcionado incremento de muertes por sobredosis de opioides asociados a benzodiazepinas (NIDA, 2017).

El Centro de Prevención y Control de Enfermedades de Estados Unidos recomienda facilitar naloxona a los pacientes que toman analgésicos opioides asociados a benzodiazepinas, o bien si tienen antecedentes de abuso de alcohol o drogas, ya que son los que tienen una mayor riesgo de sobredosis (CDC, 2016).

Cuando una persona ha sufrido una primera sobredosis por opioides debería iniciar tratamiento especializado de su trastorno por consumo de opioides inmediatamente, ya que la adicción es una enfermedad que aumenta el riesgo de sobredosis y que, por tanto, es potencialmente mortal. El tratamiento especializado, tras una primera sobredosis, puede prevenir su futura muerte accidental. Sin embargo, no es probable que la persona consumidora de analgésicos opioides reciba el diagnóstico de trastorno por consumo de opioides y con frecuencia se siguen prescribiendo opioides, a pesar de que una persona haya sufrido una sobredosis (Madras, 2017).

En Europa la sobredosis es la principal causa de muerte entre los consumidores de drogas de alto riesgo. El $78 \%$ son hombres, con un aumento de los de más edad entre 2007 y 2015 y una disminución del grupo de los más jóve- 
nes, lo cual refleja el envejecimiento de la población consumidora de opioides. En 2015 se produjeron 8441 muertes por sobredosis asociadas a alguna droga ilegal, lo cual representa un aumento del $6 \%$ con respecto a 2014. En España se ha producido también un incremento de muertes por sobredosis entre 2012 y 2015 (EMCDDA, 2017).

El sistema sanitario público español presenta algunas ventajas que podrían ayudar a prevenir una futura epidemia de analgésicos opioides y de sobredosis. La conexión informática de los servicios asistenciales de los hospitales con la atención primaria de salud y los centros de salud mental y de drogodependencias, permite el acceso a la historia clínica unificada, lo cual facilita la coordinación de los diversos médicos. La receta electrónica, a la cual tienen acceso todos los médicos del servicio público de salud, evita duplicidades y facilita la supervisión y el control de las pautas de prescripción de medicamentos. El registro informatizado sobre la prescripción de metadona es visible también para cualquier médico de la red de centros de drogodependencias. El acceso de las personas que presentan adicción a sustancias, a los centros de tratamiento de la red de drogodependencias, facilita el tratamiento especializado de su adicción. Finalmente, el acceso gratuito de toda la población, al sistema público de salud, ofrece un tratamiento simultáneo, no sólo de la adicción sino también de las comorbilidades médicas y psiquiátricas que pueda presentar la persona que sufre una adicción.

Sin embargo, puede haber una cierta banalización y desconocimiento con respecto a los riesgos de la co-prescripción y del policonsumo de medicamentos, que aumentan el riesgo de accidentes y sobredosis. Por ejemplo, en algunos hospitales se administra fácilmente morfina intravenosa "a demanda", cuando el paciente aqueja dolor intenso. Esta pauta de administración de opioides, que se puede mantener durante muchos días de hospitalización y a veces hasta que el paciente sale del hospital, conlleva un mayor riesgo de adicción, sobretodo para las personas que ya tienen antecedentes de otras conductas adictivas o bien una mayor vulnerabilidad hacia ellas.

Existe también un grave desconocimiento sobre la adicción, como una enfermedad común, que puede afectar a cualquier persona y que requiere un tratamiento especializado. Con frecuencia dicho tratamiento requiere fármacos específicos, pero algunos no son financiados por el sistema público de salud y otros presentan diversas dificultades para su prescripción o su adquisición, sobretodo cuando su adquisición resulta gravosa para el enfermo o cuando el medicamento tiene un elevado precio.

Otros factores de riesgo de sobredosis mortales, que acechan en nuestros medios y que podrían emerger en los próximos años, son la prescripción de opioides sintéticos de gran potencia -como fentanilo y derivados- y la co-prescripción de analgésicos opioides y benzodiazepinas. El consumo de benzodiazepinas es muy elevado en nuestros medios. Afecta al 15,3\% de la mujeres y 7,6\% de los hombres, aumenta con la edad y llega a ser del $27 \%$ de la mujeres y $11,3 \%$ de los hombres, en la franja de edades entre los 55 y los 65 años (Ministerio de Sanidad, Servicios Sociales e Igualdad. 2013).

\section{Prevención}

El tratamiento del dolor con analgésicos opioides a largo plazo conlleva el riesgo de que el paciente desarrolle tolerancia, con necesidad de aumentar las dosis, hiperalgesia y adicción. Su seguimiento y monitorización permite la detección de posibles señales de abuso o mal uso, cuando el paciente no sigue bien la prescripción establecida.

En 2015 el Departamento de Salud y Servicios Sociales de Estados Unidos ha iniciado una campaña para reducir la prescripción de analgésicos opioides y las muertes por sobredosis, mediante tres estrategias: (1) entrenamiento y formación de los médicos sobre tratamiento del dolor para reducir la prescripción de opioides, (2) facilitar el acceso a naloxona y (3) expandir la disponibilidad de tratamiento de la adicción con medicamentos opioides, que incluya también a los menores de edad (Dowell, Haegerich, y Chou, 2016).

Han desarrollado un programa electrónico para monitorizar las prescripciones de opioides y promover las prácticas de prescripción seguras. El CDC ha desarrollado un Guía para la prescripción de opioides con las siguientes recomendaciones: (1) limitar el inicio del abuso y adicción a los analgésicos opioides mediante la instrucción de los médicos, (2) expandir los tratamientos con medicamentos (basados en la evidencia) para las personas que han desarrollado adicción a opioides, (3) proteger a las personas que tienen adicción a opioides con un fácil acceso a la naloxona en caso de sobredosis y (4) coordinar las acciones de todos los profesionales que les atienden para optimizar la detección y la respuesta terapéutica de las personas que han sufrido alguna sobredosis (CDC, 2016).

Otras recomendaciones son la preferencia de los analgésicos corrientes para el tratamiento del dolor crónico, la restricción en la prescripción de analgésicos opioides a aquellos casos en los que los beneficios sobre el dolor y la funcionalidad sean superiores a los riesgos asociados, establecer objetivos de tratamiento con los pacientes y considerar previamente la posibilidad de retirarlos si los beneficios no superan a los riesgos, prescribir la dosis mínima eficaz, reevaluar cuidadosamente beneficios y riesgos si están pensando superar la dosis de $50 \mathrm{mg}$ / día de morfina o equivalentes y evitar la prescripción y el consumo simultáneo de otros opioides o de benzodiazepinas, siempre que sea posible. También deberían introducir los datos de prescripción en un programa electrónico que advierta de posibles dosis o combinaciones de riesgo. Y para las personas que hayan desarrollado adicción a opioides, ofrecer y facilitar 
el acceso a programas de tratamiento especializados y con medicamentos como buprenorfina o metadona (Dowell, et al., 2016).

La adicción a opioides es una enfermedad del cerebro que puede ser tratada eficazmente, aunque a veces se requieren varios episodios de tratamiento para que el paciente se recupere por completo. Su tratamiento requiere la administración de fármacos agonistas de los receptores opioides, como metadona o buprenorfina (NIDA, 2012).

Conviene sopesar también si los beneficios de los analgésicos opioides son sólo pequeños o moderados a corto plazo e inciertos a largo plazo, en comparación con sus potenciales graves efectos adversos. Por ejemplo, para el tratamiento de la cefalea o la fibromialgia, los beneficios esperables de los opioides probablemente no sobrepasan los riesgos potenciales, mientras que los analgésicos corrientes, asociados a antidepresivos y/o anticonvulsivantes, pueden ser incluso más eficaces y menos arriesgados. Ninguna evidencia demuestra que los analgésicos opioides tengan beneficio a largo plazo para el dolor y, sin embargo, muchas evidencias demuestran sus posibles consecuencias negativas, como adicción, sobredosis y lesiones por accidentes de tráfico, mientras que algunas evidencias sugieren ciertos beneficios de tratamientos no farmacológicos, que además son más inocuos (Dowell et al., 2016).

En el tratamiento del dolor agudo intenso, los opioides pueden ser necesarios para fracturas óseas, cólico nefrítico, infarto de miocardio y otros. Sin embargo, para dolores de menor intensidad como lumbalgia, cefalea, fibromialgia o dolor dentario, los analgésicos corrientes, el reposo y la fisioterapia pueden ser incluso más eficaces que los opiáceos (Lligoña, López, Henche, Guardia, Tuca, et al., 2017).

En el dolor crónico no deberían ser considerados como tratamiento de rutina o de primera línea, con la excepción del cáncer activo, cuidados paliativos o enfermos terminales. Conviene evitar la prescripción indiscriminada de analgésicos opioides siempre que sea posible y como primera opción es preferible utilizar analgésicos corrientes, que pueden ser asociados a fármacos coadyuvantes como anticomiciales y antidepresivos (Guardia Serecigni, 2017).

Se ha recomendado también advertir a los pacientes sobre los efectos adversos de los opioides, como estreñimiento, sequedad de boca, náuseas, vómitos, somnolencia, confusión, tolerancia, dependencia física y síntomas de abstinencia, cuando se interrumpe su administración. Y también sobre su interferencia en la capacidad para conducir con seguridad, sobretodo al inicio del tratamiento, cuando se produce un aumento de dosis o cuado se toman a la vez otros depresores como ansiolíticos, hipnóticos o bebidas alcohólicas (CDC, 2016).

Dado que Estados Unidos está padeciendo una epidemia de sobredosis, debido a una etapa de prescripción indiscriminada de fármacos analgésicos opioides y dado que todavía estamos a tiempo de prevenir una epidemia parecida en nuestro país, consideramos apropiada la divulgación de esta información, no sólo a los profesionales de la medicina sino también a toda la población, ya que entre todos podemos contribuir a evitarlo.

\section{Conflicto de Intereses}

El autor ha recibido una ayuda económica del Laboratorio Lundbeck, para efectuar un estudio sobre la reducción del consumo de alcohol con nalmefeno, que no tiene ninguna relación con este Editorial.

\section{Referencias}

Case, A. y Deaton, A. (2015). Rising morbidity and mortality in midlife among White non-Hispanic Americans in the 21st Century. Proceedings of the National Academy of Sciences of the United States of America, 112, 15078-15083. doi: $10.1073 /$ pnas. 1518393112

Brauser, D. (2017). NEJM Letter the Genesis of the Opioid Crisis? Medscape, Jun 06. Recuperado de https://www. medscape.com/viewarticle/881215_print.

CDC (2016). CDC guidelines for prescribing opioids for chronic pain. Centers for Disease Control and Prevention. Recuperado de http://www.cdc.gov/drugoverdose/prescribing/resources.hrml.

Chen, L., Hedegaard, H. y Warner, M. (2014). Drug-poisoning deaths involving opioid analgesics: United States, 1999-2011. NCHS Data Brief, no. 166. Hyattsville, MD: National Center for Health Statistics.

Corkery, J. M., Schifano, F., Ghodse, A. H. y Oyefeso, A. (2004). The effects of methadone and its role in fatalities. Human Psychopharmacology, 19, 565-576. doi:101002/hup.630.

Dowell, D., Haegerich, T. M. y Chou, R. (2016). CDC Guidelines for prescribing opioids for chronic pain-United States, 2016. Journal of American Medical Association, 315, 1624-1645. doi:10.1001/jama.2016.1464.

EMCDDA (2017). Informe Europeo sobre Drogas. Tendencias y novedades. Lisboa: Observatorio Europeo de las Drogas y las Toxicomanías. Oficina de publicaciones de la Unión Europea.

Guardia, J., Surkov, S. y Cardús, M. (2010). Neurobiología de la adicción. En C. Pereiro Gómez (Ed.), Manual de adicciones para médicos especialistas en formación (pp. 37130). Barcelona: Socidrogalcohol.

Guardia, J. (2017). Introducción. En J. Guardia Serecigni, A. Henche Ruiz y A. Lligoña Garreta (Eds.), Guía de consenso sobre el buen uso de analgésicos opioides. Gestión de riesgos y beneficios (pp. 127-137). Valencia: Socidrogalcohol.

Hikin, L., Smith, P. R., Ringland, E., Hudson, S. y Morley, S. R. (2017). Multiple fatalities in the North of England associated with synthetic fentanyl analogue exposure: De- 
tection and quantitation a case series from early 2017. Forensic Science International, 282, 179-183. doi:10.1016/j. forsciint.2017.11.036.

Horsfall, J. T. y Sprague, J. E. (2016). The pharmacology and toxicology of the 'Holy Trinity'. Basic and Clinical Parmacology E Toxicology, 120, 115-119. doi:10.1111/ bcpt. 12655 .

Hwang, C. S., Kang, E. M., Kornegay, C. J., Staffa, J. A., Jones, Ch. M. y McAninch, J. K. (2016). Trends in the concomitant prescribing of opioids and benzodiazepines, 2002-2014. American Journal of Preventive Medicine, 51, 151-160. doi:10.1016/j.amepre.2016.02.014.

Jones, C. M. y McAninch, J. K. (2015). Emergency department visits and overdose deaths from combined use of opioids and benzodiazepines. American Journal of Preventive Medicine, 49, 493-501. doi:10.1016/j.amepre.2015.03.040.

Kalivas, P. W. y Volkow, N. D. (2005). The neural basis of addiction: a pathology of motivation and choice. American Journal of Psychiatry, 162, 1403-1413. doi:10.1176/appi. ajp.162.8.1403.

Leung, P. T. M., Macdonald, E. M., Stanbrook, M. B., Dhalla, I. A. y Jauurlink, D. N. (2017). A 1980 Letter on the Risk of Opioid Addiction. New England Journal of Medicine, 376, 22. doi:10.1056/NEJMc1700150.

Lin, K. W. y Vega, Ch. P. (2016). Navigating the opioid crisis: a primary care perspective. Department of Family Medicine. Georgetown University Medical Center. Medscape, Aug 02. Recuperado de http://www.medscape. com/viewarticle/866579.

Lligoña, A., López, A., Henche, A. I., Guardia, J., Tuca, A., Sánchez, C.,... Pascual, P. (2017). Recomendaciones para un uso racional y seguro de los analgésicos opioides. En J. Guardia Serecigni, A. Henche Ruiz y A. Lligoña Garreta (Eds.), Guía de consenso sobre el buen uso de analgésicos opioides. Gestión de riesgos y beneficios (pp. 127137). Valencia: Socidrogalcohol.

Madras, B. (2017). The surge of opioid abuse, addiction and overdoses. Responsibility and response of the US Health Care System. Journal of American Medical Association Psychiatry, 74, 441-442. doi:10.1001/jamapsychiatry.2017.0163.

Ministerio de Sanidad, Servicios Sociales e Igualdad (2013). Estudio EDADES 2011-2012. Madrid: Ministerio de Sanidad, Servicios Sociales e Igualdad. Secretaría de Estado de Servicios Sociales e Igualdad. Delegación del Gobierno para el Plan Nacional sobre Drogas.

NIDA. National Institute on Drug Abuse. (2012). Cómo prevenir y reconocer el abuso de medicamentos de prescripción. En Español. Serie de Reportes de Investigación. Departamento de Salud y Servicios Humanos de los Estados Unidos. Institutos Nacionales de la Salud.

NIDA. National Institute on Drug Abuse. (2017). Overdose death rates. Revised September 2017. Recuperado de ht- tps://www.drugabuse.gov/related-topics/trends-statistics/overdose-death-rates.

O’Donnell, J. K., Halpin, J., Mattson, Ch. L., Goldberger, B. A. y Gladen, M. (2017). Deaths involving fentanyl analogs, and U-47700 - 10 States, July-December 2016. Morbidity and Mortality Weekly Report, 66, 1197-1202. doi:10.15585/mmwr.mm6643e1.

Olfson, M., Wall, M., Wang, S., Crystal, S. y Blanco, C. (2017). Service use preceding opioid-related fatality. American Journal of Psychiatry. Avance de publicación on-line. doi:10.1176/appi.ajp.2017.17070808.

Park, T. W., Saitz, R., Ganoczy, D., Ilgen, M. A. y Bohnert, A. B. (2015). Benzodiazepine prescribing patterns and deaths from overdose among US veterans receiving opioid analgesics: case-cohort study. British Medical Journal, 350, h2698. doi:10.1136/bmj.h2698.

Paulozzi, L. J., Jones, Ch. M., Marck, K. A. y Rudd, R. A. (2011). Vital signs: overdoses of prescription opioid pain relievers-United States, 1999-2008. Morbidity and Mortality Weekly Report, 60, 1487-1492.

Rudd, R. A., Seth, P., David, F. y Scholl, L. (2016). Increases in drug and opioid involved overdose deaths - United States, 2010-2015. Morbidity and Mortality Weekly Report, 65, 1445-1452. doi:10.15585/mmwr.mm655051e1.

SAMHSA. Substance Abuse and Mental Health Services Administration. (2014). The DAWN Report: Benzodiazepines in Combination With Opioid Pain Relievers or Alcohol: Greater Risk for More Serious ED Visit Outcomes (pp. 1-6). Rockville, MD: US Department of Health and Human Services, Substance Abuse and Mental Health Services Administration. Center for Behavioral Health Statistics and Quality. 